\title{
LXII. A simple resonance experiment
}

\section{O. Steels}

To cite this article: O. Steels (1915) LXII. A simple resonance experiment, Philosophical Magazine Series 6, 30:178, 623-627, DOI: 10.1080/14786441008635434

To link to this article: http://dx.doi.org/10.1080/14786441008635434

册 Published online: 08 Apr 2009.

Submit your article to this journal

Џ Article views: 2

Q View related articles $₫$ 


\section{Conclusion.}

The investigation in this paper has been largely restricted to a consideration of the various modes in which compound molecules of the two elements hydrogen and carbon may be formed. It will be evident to those who have followed the processes that, when some of the other kinds of atoms are introduced, the line of investigation will be greatly extended, and seems likely to have important practical consequences. Some of these questions have already been considered, but it must be borne in mind that before exact results can be obtained by this method the charts for the fields of force for each pair of atoms entering the compound molecule must be worked out. To add the single element oxygen, for example, requires three more charts, one for oxygen on oxygen, a second for oxygen on hydrogen, and a third for oxygen on carbon, and these charts are not yet available.

LXII. A Simple Resonance Experiment. By O. STEELs, Professor of Applied Electricity in the University of Ghent (Belgium), Chief-Engineer and Director in the Belgian Telegraphic Department*

1. SINCE resonance phenomena play an important part

$\checkmark$ in several physical questions, it is of some interest, especially for lecture purposes, to devise a simple experiment to illustrate this phenomenon, and to bring it within the grasp of common knowledge and observation.

Text-books have described devices making use of pendulums. In the Jalrbuch der drahtlose Telegraphie, 1912, we find the theory of a mechanical arrangement, the equations for which are similar to those arising in the case of the electrical resonance of two coupled electrical circuits excited by a third one.

In the simple case of two electrical circuits loosely coupled, we know that it is easy to plot a resonance curve making use, for instance, of some wavemeter; but the experiment, though not difficult, is not very striking.

For this reason it is advisable to arrange an eleetromechanical combination, making use of apparatus to be found in any laboratory, and which is consequently easy to put together.

2. Let us take a little magnet $\mathrm{N}$-for instance, one of those little models which are used for tracing the force-lines

* Communicated by Prof. A. W. Porter, F.R.S. 
of magnetic fields-in the neighbourhood of a coil $B$ connected to the rings $C$ of a bipolar commutator $D$ running at a constant velocity. Close to the milgnet we place an electromagnet-an iron rod $\mathrm{D}$ surrounded by a coil $\mathrm{A}-$ connected to a battery, the current being regulated by the rheostat $R$ and read on the ammeter A, fig. 1 .

Fig. 1.

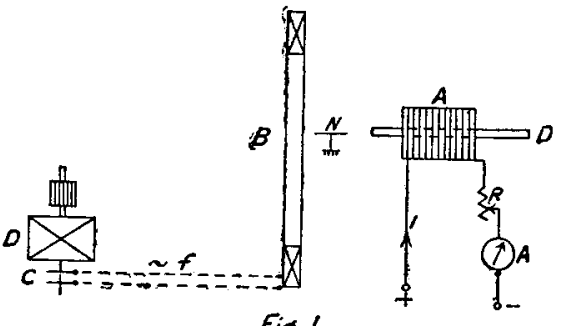

Fig. I

If we consider either the positions of the electromagnet $A$ shown in fig. 2 or in fig. 3, we observe that the needle $\mathrm{N}$ is

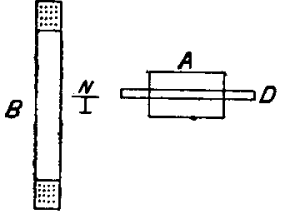

Fig.2

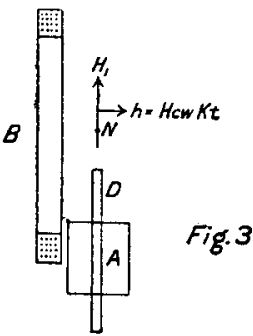

Fig. 2.

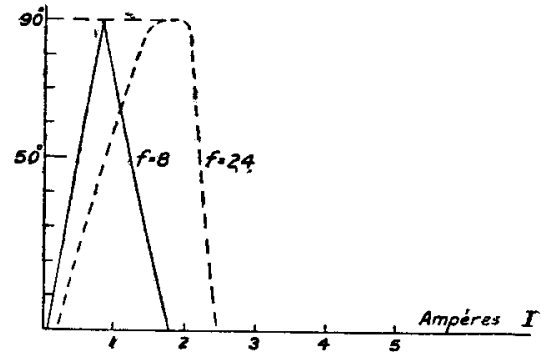

Fig. 3,

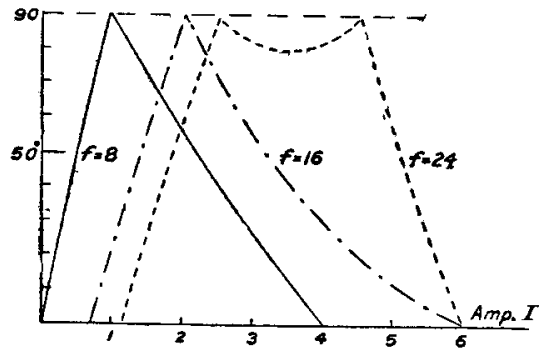

at rest when the current $I$ is cut off. If we increase this current, we see the needle starting a periodical vibration the amplitude of which rises with the magnitude of the current I, passes through a maximum, and decreases till, for a sufficiently large current $I$, the needle is again at rest. 
We may proceed in another way. Taking a suitable value for the current I which we maintain constant, we slowly remove the iron bar $\mathrm{D}$ from the electromagnet, and we observe, as above, the same variable periodic movement of the magnetic needle, which illustrates in a striking manner the phenomena known under the general name of resonance.

3. If we do not insist on accuracy, it is possible to estimate the amplitude of the vibration of the needle for different values given to the current $I$, and plot resonance curves for different values of the frequency of the current flowing through the field-coil B. For different experiments we can alter also the relative positions of the needle $\mathrm{N}$ and the electromagnet $A$ as is shown in fig. 2 and fig. 3. Fig. 2, for instance, gives the resonance curves for the relative positions sketched alongside, and frequencies 8 and 24 for the current flowing through the coil B. Fig. 3 gives, similarly, curves for the positions sketched alongside, and for $f=8,16$, and 24 .

4. In order to be able to justify the shapes of these curves it would be necessary to go into the mathematical theory of the phenomena, which is a very elaborate one. We shall only consider a particular case, for the mathematical treatment of which we suppose the relative positions of the different fields given in fig. 3. We suppose that the magnet is reduced to its two poles, and is of magnetic moment $M$, that its oscillations have only a small amplitude, that the resultant field $\mathrm{H}_{1}$ produced by the electromagnet and the earth is constant and at right angies to the field $h=\mathrm{H} \cos k t$ produced by the coil.

If we suppose $h=0$, the needle is able to oscillate when disturbed out of its normal position. If there is no damping, the deviation angles correspond to the equations

$$
\alpha=\mathrm{A} \sin k_{1} t, \quad k_{1}=2 \pi f_{1},
$$

the frequency $f_{1}$ being given by the relation

$$
f_{1}=\frac{1}{2 \pi} \sqrt{\frac{\overline{\mathrm{M} \cdot \mathrm{H}_{1}}}{\mathrm{~K}}}
$$

$\mathrm{K}$ being the moment of inertia of the needle. In increasing $\mathrm{H}_{1}$ or the current I we increase $f_{1}$.

If we consider damping exists, the equation of the movement becomes

$$
\frac{d^{2} \alpha}{d t^{2}}+n^{2} \alpha+2 p \frac{d \alpha}{d t}=0
$$

and can be studied graphically by means of the kinematic Phil. Mag. S. 6. Vol. 30. No. 178. Oct. 1915. 2 S 
representation of 'lait, making use of the properties of the logarithmic spiral of Descartes,

If we now apply the alternating field $h=\mathrm{H} \cos k t$, the equation becomes

$$
\frac{d^{2} \alpha}{d t^{2}}+n^{2} \alpha+2 p \frac{d \alpha}{d t}=\mathrm{E} \cos k t
$$

which is satisfied by a solution of the form

$$
\alpha=\mathrm{F} \sin (k t+\phi) \text {, called a forced vibration. }
$$

Its superposition upon the free damped vibration of the system gives the general integral, and the classical study of the case leads to the conclusion that resunance occurs, the power of the external vibrating force being maximum at that moment when the frequency constant $k$ is equal to the value of $k_{1}, i$.e the value of the frequency constant of the free vibration when no friction is present.

If we refer to figs. 2 and 3 we now see the reason why the resonance curves shift to the right when the frequency of the alternating current increases; for when $f$ the frequency of the current flowing through becomes larger, we must increase also $f_{1}$ and consequently $I$ in order to obtain resonance.

The curves for the high values of the frequency are flatter than those for the lower frequencies, and curve No. 3 shows even two maximum values, the first corresponding to the oscillations in which on $\theta$ of the poles of the needle points towards the $\mathrm{N}$ pole of the solenoid, and the second those in which the other pole of the needle points towards the same pole of the solenoid.

5. The value of the current I giving the resonance phenomena can be checked very accurately.

For that purpose we notice that our experimental arrangement is a synchronous monophase motor with variable reluctance and reduced to its theoretical simplicity. We suppose the current I shut off. The theory of that kind of alternate motors was given by Blondel, and I applied Blondel's theory to explain in a more adequate manner than is usually done the process of starting the ordinary synchronous motor *. The peculiar qualities of that kind of motors are well known; the very simple experimental arrangemont we have before us gives us an opportunity of verifying some of them in a very easy way. First we observe that the rotor (the needle) being at rest, there is no starting

* Revue Electrique, Paris, 1912. 
moment : the needle remains at rest. In order to put the rotor in action we inust give it an impulse, and the starting rotation can be brought up to the value for synchronism, the rotor being, as we say in French, "accrochée," by a physical process I established in the paper already referred to.

Now, we shall obtain the best conditions for having a suitable impulse by bringing first the rotor into resonance with the help of the auxiliary field. Cutting off at that given moment the direct current, wo observe that the needle turns round, and if we illuminate it with a lamp put on the main alternating current, we can observe in the dark that its shadow is at rest, and verify thus by the ordinary stroboscopic method that the rotor is turning at the synchronous speed, and is "accroché." We can now remove if we like the electromagnet $A$ and the rod $D$. If we move a magnet near the needle we can bring it again to rest, and thus verify another law of the synchronous motor: it falls out of step when the resistant moment becomes too large.

6 . If we desire to show the resonance experiment rougbly - for example during a lecture-the most expeditious way is to raise the current $I$ to such a value that the needle is quite motionless for a given position of the iron rod. We then pull the iron rod back with a slow motion. At a given moment we shall observe a maximum deflexion of the needle, and if we continue to move the iron rod, we observe that the vibration of the needle decreases in amplitude, and the needle comes again to rest.

The experiment can be performed by making use of needles of different inertia and appropriate frequencies of the alternating current.

Oxford, Prof. Townsend's Laboratory. July 1915.

LXIII. The Decrease in Velocity of $\beta$-partioles in passing through Matter. By W. F. Rawlinson, M.Sc.*

$7 \mathrm{HE}$ decrease in velocity of $\beta$-particles in traversing matter is difficult to measure on account of the marked scattering which they suffer. W. Wilson $\dagger$ examined the problem in detail by an ionization method; but as bis initial beam was not homogeneous, his final results prove only that there is a reduction in the average velocity of $\beta$-rays in

* Communicated by Sir E. Rutherford, F.R.S.

† Proc, Roy. Soc. A. lxxxiv, p. 141 (1910). 